\title{
Fibrosis quística: aumento de la sobrevida en un centro especializado a 10 años de seguimiento
}

\author{
EDUARDO LENTINI N. ${ }^{1}$, ADRIANA LÓPEZ-MILLÁN. ${ }^{1}$, ANA MARÍA LORES L. ${ }^{1}$, \\ MARÍA ROSA PESCIULLESI B. ${ }^{1}$, LEONOR MARÍA ITUARTE R. ${ }^{2}$, TERESA BEATRIZ VIERA C. ${ }^{2}$ \\ 1. Centro de Fibrosis Quística, Hospital Pediátrico Dr. H. J. Notti, Mendoza, Argentina. \\ 2. Universidad Nacional de Cuyo, Facultad de Ciencias Médicas, Cátedra de Física-Biológica, Argentina.
}

\begin{abstract}
Improving cystic fibrosis survival as a result of management in a specialized Center with 10-year follow-up

Introduction: Cystic fibrosis (CF), the most common genetic disease among whites, is a multisystem disease that requires a coordinated multidisciplinary approach to get better results regarding survival and quality of life. This is why a Cystic Fibrosis Center (CFC) was held in Mendoza, Argentina, with the assumption that the organization and management of $\mathrm{CF}$, according to international standards would improve survival. Patients and Method: 106 patients with CF have been evaluated since 1975 in this Center. Patients were grouped for follow-up monitoring as the 1999, 2002, 2005 and 2008 cohort studies. The chi-square test was used to compare survival rates and the nonparametric Mann-Whitney test was used to calculate the median age of the population. Results: In 2008, survival at 17 years old was $80 \%$ compared with $45 \%$ for the same age in 1999 ( $\mathrm{p}<0,001)$. The median age of the total CF population increased from 4 (1985) to 12 years old (2008) $(\mathrm{p}<0,003)$. Conclusions: A multidisciplinary standardized approach of this condition in a CF Center can significantly increase survival.
\end{abstract}

(Key words: Cystic fibrosis, Cystic Fibrosis Center, survival, mortality).

Rev Chil Pediatr 2014; 85 (3): 281-287

\section{RESUMEN}

Introducción: La Fibrosis Quística (FQ), la enfermedad genética más frecuente de la raza blanca, es una patología multisistémica y como tal requiere un abordaje multidisciplinario coordinado para obtener mejores resultados de sobrevida y calidad de vida. Con este propósito se organizó un Centro de Fibrosis Quística (CDFQ) en Mendoza, Argentina, y se diseñó este estudio con el objetivo de evaluar la hipótesis de que la organización y tratamiento de la FQ de acuerdo con normas internacionales, mejora la sobrevida. Pacientes y Método: Se evaluaron 106 pacientes con FQ a partir del año 1975 en el CDFQ. Los pacientes se agruparon para su seguimiento como cohortes de los años 1999, 2002, 2005 y 2008. Para comparar tasas de sobrevida

Recibido el 25 de enero de 2013, devuelto para corregir el 06 de septiembre de 2013, segunda versión 29 de octubre de 2013 , aceptado para publicación el 07 de febrero de 2014.

Este trabajo cumple con los requisitos sobre consentimiento /asentimiento informado, comité de ética, financiamiento, estudios animales y sobre la ausencia de conflictos de intereses según corresponda.

Correspondencia a:

Dr. Eduardo Lentini N.

E-mail: doclentini@gmail.com 
se usó el test de $\chi^{2}$ y para edad mediana de la población se usó el test no paramétrico de Mann-Whitney. Resultados: En el año 2008 la sobrevida a los 17 años fue de 80\% comparado con el año 1999 donde esta cifra alcanzó $45 \%$ a la misma edad ( $<<0,001)$. La mediana de edad de la población con FQ total aumentó de 4 años (1985) a 12 años (2008) $(\mathrm{p}<0,003)$. Conclusiones: Un enfoque multidisciplinario y normatizado en un CDFQ puede aumentar la sobrevida significativamente.

(Palabras clave: Fibrosis quística, Centro de Fibrosis Quística, sobrevida, mortalidad).

Rev Chil Pediatr 2014; 85 (3): 281-287

\section{Introducción}

Existen importantes disparidades en los resultados de sobrevida en Fibrosis Quística (FQ) cuando se compara el mundo en desarrollo con el mundo desarrollado, el cual muestra cifras de sobrevida extendiéndose hasta la cuarta década de vida ${ }^{1-3}$. Estos resultados se han atribuido, entre otros, a los siguientes factores: atención en Centros de Fibrosis Quística $(\mathrm{CDFQ})^{4}$; disponibilidad y uso de antibióticos contra Pseudomonas aeruginosa (PA) ${ }^{5,6}$; mejor manejo nutricional incluyendo enzimas pancreáticas ${ }^{7}$; uso de ADNasa recombinante ${ }^{8}$, diagnóstico precoz por screening neonatal y técnicas modernas de estudio de vía aérea menor ${ }^{9,10}$. Los factores asociados a peores resultados en Latinoamérica ${ }^{11}$ incluyen diferencias socio-económicas, acceso desigual a la salud, bajos presupuestos de salud y la carencia de organizaciones focalizadas en la FQ.

En el CDFQ se han introducido instrumentos de detección, confirmación diagnóstica y estrategias terapéuticas novedosas. Dentro de los primeros, se ha implementado por ley en nuestra Provincia el mapeo neonatal con tripsinógeno inmunorreactivo (TIR). Su evaluación y confirmación diagnóstica en recién nacidos en maternidades estatales está centralizada en el CDFQ. La confirmación diagnóstica se hace por el test del sudor (técnica de Gibson y Cook $)^{12}$ y/o estudios genéticos. Se han incorporado protocolos de tratamiento de la Cystic Fibrosis Foundation (EEUU) en diversas áreas incluyendo desarrollo de las técnicas de colocación y cuidado de catéteres centrales de inserción periférica ${ }^{13}$, uso de bombas de infusión, cambio a antibióticos de nueva generación de acuerdo con los cultivos microbiológicos ${ }^{14}$, tratamiento agresivo de la desnutrición con alimentación por gastrostomía, entrenamiento de los pacientes en nuevas técnicas de kinesiología respiratoria ${ }^{15}$, tratamiento de ADNasa, Tobramicina y Colistina inhaladas ${ }^{16}$, uso de azitromicina a largo plazo, apoyo con ventilación no invasiva a pacientes debilitados, desarrollo de un sistema estatal para tratamiento domiciliario, y la existencia de un Centro de Trasplantes cercano al que se derivan enfermos.

Las políticas Nacionales de Salud dependen de una autoridad Central (Ministerio de Salud) que establece políticas globales solamente en casos particulares (ie: campañas de vacunación). La mayor parte del cuidado de la salud en las Provincias depende de sus propios presupuestos y las decisiones estratégicas en salud, con importantes diferencias entre Provincias "ricas" y "pobres", con grandes contrastes en el acceso a la medicación y a organizaciones especializadas en FQ. Mendoza es una de las pocas provincias del Oeste Argentino que tiene un CDFQ organizado según normas internacionales desde 1989, con un presupuesto propio, que atiende pacientes con o sin cobertura de Obra Social. En la actualidad, el CDFQ está compuesto por un equipo multidisciplinario constituido por neumonólogos, así como una administración propia que organiza la atención, provisión de medicamentos y mantiene las bases de datos. Otros especialistas concurren al CDFQ según necesidad, para que el paciente cuente con una atención integral en un solo lugar. La atención se hace en días diferentes según estén colonizados por PA o no. Los pacientes son atendidos desde su diagnóstico, sin límites de edad, aunque las internaciones en mayores de 18 años se hacen en un hospital de adultos.

El objetivo de este estudio fue evaluar la hi- 
pótesis de que la organización y tratamiento de la FQ de acuerdo con normas internacionales en un CDFQ mejora la sobrevida.

\section{Pacientes y Método}

\section{Diseño del estudio}

Estudio retrospectivo de sobrevida basado en revisión de historias clínicas, durante 10 años, en una población de pacientes con FQ del CDFQ de Mendoza, Argentina. La población total de pacientes estudiada fue de 106 pacientes, divididos en cohortes para su análisis. Los criterios de inclusión fueron los de la Cystic Fibrosis Foundation ${ }^{17}$.

\section{Conformación de las cohortes}

Los pacientes incluidos inicialmente como la cohorte de 1999 fueron todos aquellos con diagnóstico de FQ desde el $1^{\circ}$ de enero de 1975 hasta el 31 de diciembre de 1999 (primera cohorte). Se siguió estudiando a estos pacientes como las cohortes de 1999, 2002, 2005 y 2008, agregando nuevos diagnósticos y censurando los datos de los perdidos al seguimiento y dando por terminado el seguimiento de los fallecidos. Los pacientes fueron evaluados desde el momento del diagnóstico (visita a consultorio externo y confirmación diagnóstica) hasta el momento en que mueren o no concurren al CDFQ (perdidos al seguimiento). Los pacientes sobrevivientes se incluyeron en las cohortes anteriormente detalladas. Los números de pacientes en cada cohorte fueron: 1999: $\mathrm{n}=$ $55 ; 2002: \mathrm{n}=63 ; 2005: \mathrm{n}=79 ; 2008: \mathrm{n}=88$.

\section{Seguimiento}

Durante este período de seguimiento cada paciente fue considerado individualmente. Éste puede fallecer, perderse al seguimiento o estar vivo en el momento en que el estudio de cada cohorte terminó. El tiempo de sobrevida se definió como los años que transcurrieron desde el tiempo 0 (momento del diagnóstico cuando ingresó al CDFQ) hasta el momento que muere. La probabilidad de sobrevida en cualquier momento del tiempo se calculó sobre la probabilidad acumulada de sobrevivir en cada período de tiempo precedente. Aunque el seguimiento de cada paciente comenzó en diferentes fechas del estudio, la metodología aplicada consideró esto, y por lo tanto, el momento del ingreso al estudio no influenció los resultados.

\section{Fuentes de datos}

Las historias clínicas fueron sistemáticamente revisadas por dos investigadores que obtuvieron: edad al diagnóstico, género, colonización por PA, colonización por $S$. aureus, situación socio-económica, nutrición y edad al fallecer.

\section{Definiciones}

Los pacientes perdidos al seguimiento fueron aquellos que no concurrieron durante 1 año a la consulta aún después de tres llamados telefónicos. Los pacientes colonizados por PA fueron aquellos con tres cultivos anuales positivos para PA. Colonización por $S$. aureus se consideró a aquellos con tres cultivos anuales positivos. La desnutrición se definió con un índice de masa corporal $<10$.

\section{Análisis estadístico}

Para comparar las tasas de sobrevida para los años 1999, 2002, 2005 y 2008 se usó el test de chi-cuadrado $\left(\chi^{2}\right)$. Para la edad mediana de la población usó un test no-paramétrico (Mann-Whitney). Para determinar la significancia de la edad mediana de los pacientes sobrevivientes de las cohortes de 1999-2008 se usó el test de Wilcoxon.

\section{Aspectos éticos}

Este trabajo se ha hecho manteniendo la confidencialidad de los datos referidos a los pacientes y cuenta con la aprobación del Comité de Ética Hospitalario.

\section{Resultados}

\section{Características demográficas de los pacientes}

El 13,6\% de nuestros pacientes tuvo más de 15 años y el $25 \%$ más de 18 años. El rango de edades fue de 4 meses a 36 años. El 68,8\% fueron hombres y el 31,3\% mujeres (tabla 1). El $50 \%$ de los pacientes se encontró colonizado por PA. La colonización por $S$. aureus mostró meticilino resistencia en $20 \%$ de los cultivos realizados y meticilinosensible en 33\% de los cultivos realizados. El $20 \%$ de los pacientes 
Tabla 1. Características demográficas de los pacientes por cohortes

\begin{tabular}{|lcccc|}
\hline Cohortes & $\mathbf{1 9 9 9}$ & $\mathbf{2 0 0 2}$ & $\mathbf{2 0 0 5}$ & $\mathbf{2 0 0 8}$ \\
Pacientes $(\mathrm{n})$ & 55 & 63 & 79 & 88 \\
Edad media (años) & 8,00 & 9,25 & 9,99 & 11,61 \\
Pacientes perdidos en seguimiento (n) & 14 & 1 & 2 & 3 \\
Edad media pacientes perdidos en seguimiento (años) & 11,4 & 36 & 16,5 & 12 \\
Mujeres-Hombres (\%) & $46-54$ & $44-56$ & $19-81$ & $32-68$ \\
Edad media al diagnóstico (años) & 3,3 & 4,18 & 3,64 & 3,33 \\
\hline
\end{tabular}

se encontraban desnutridos y en vigilancia específica. La cobertura por Obra Social sólo abarcó al 60\%; el restante $40 \%$ fueron cubiertos por el Estado Provincial. Con respecto a la condición socioeconómica, el $90 \%$ de los pacientes pertenecían a clase social de bajos ingresos; el $80 \%$ eran blancos y el $20 \%$ restante eran mestizo/amerindios.

\section{Sobrevida}

Se observó un incremento significativo de la tasa media de sobrevida durante los 10 años de seguimiento para las cohortes de los años $1999,2002,2005$ y 2008 ( $\mathrm{p} \leq 0,001)$ (figura 1$)$.

El incremento de la mediana de la edad de la población FQ fue de 4 años (1985) a 12 años (2008). Para la mediana de la edad de 4 años, el número de pacientes fue de 15 , para la mediana de la edad de 12 años el número de pacientes fue de 112 ( $\mathrm{p} \leq 0,003$ ) (figura 2). La mediana de edades para pacientes sobrevientes en cada cohorte fue en 1999 de 8 años; en 2002 de 9,25 años; en 2005 de 9,99 años y en 2008 de 11,61 años $(\mathrm{p} \leq 0,003)$.
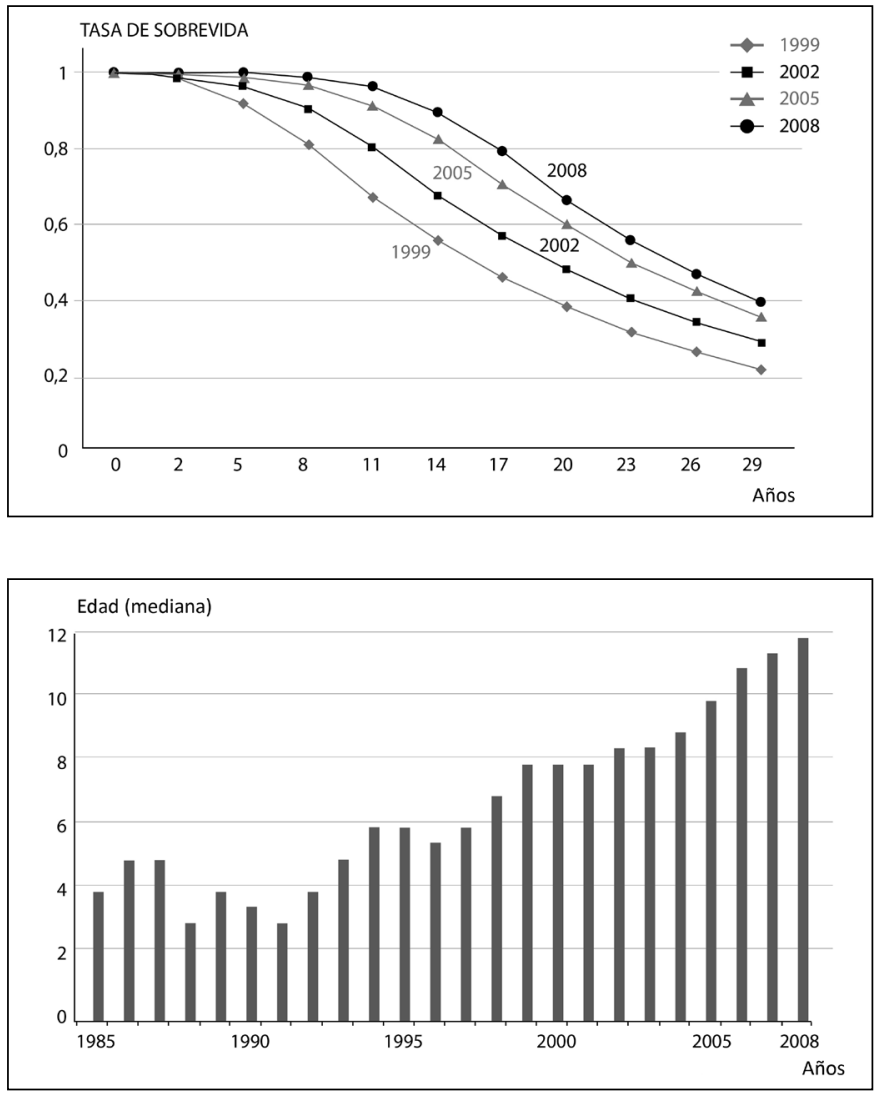

Figura 1. Tasa media de sobrevida desde 1999 a 2008. Resultados de las cohortes 1999, 2002, 2005 y 2008. Se observa aumento de la sobrevida especialmente a los 17 años de edad. Para la cohorte de 1999 la sobrevida es cercana al 45\%. Para la cohorte del año 2008 la cifra aumenta a $80 \%$ para la misma edad (17 años). Diferencia significativa, $\mathrm{p} \leq 0,001$.

Figura 2. Edad mediana de los niños FQ. La edad mediana se incrementó a partir del año 1985 hasta el año 2008. La edad mediana muestra un incremento desde los 4 años de edad en 1985 a 12 años de edad en el año 2008. 


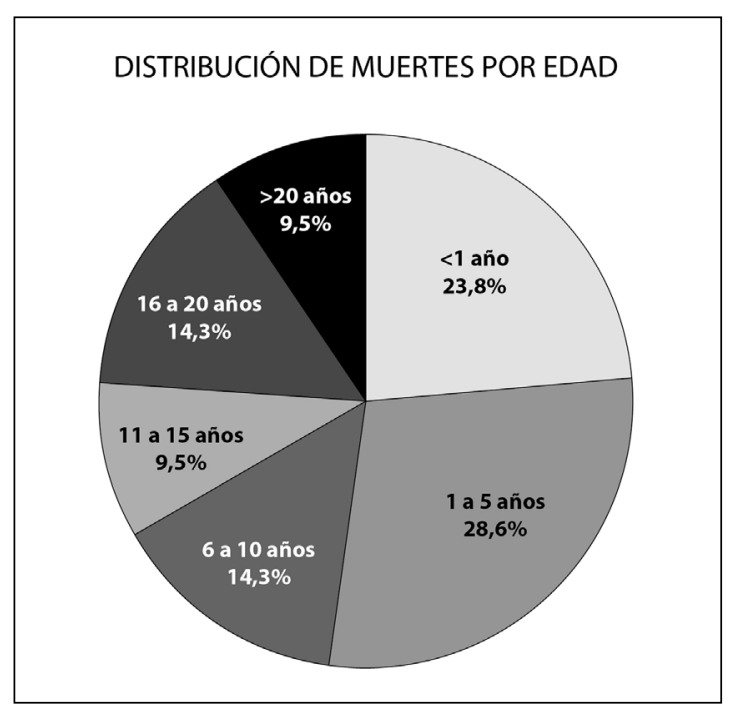

Figura 3. Mortalidad de acuerdo a rango de edad, expresada en porcentajes del total.

\section{Fallecidos}

La figura 3 muestra la distribución de porcentajes de fallecidos por rango de edad. La causa de muerte fue una falla respiratoria en todos los casos.

\section{Discusión}

En el mundo desarrollado se han observado importantes mejorías en la sobrevida de niños con FQ asociado con la organización de CDFQ. En EEUU la edad mediana de sobrevida es actualmente de 37 años ${ }^{1}$. No tenemos bases de datos epidemiológicas en FQ en América Latina para hacer comparaciones con cifras de EE.UU. o Europa. En América Latina el peor pronóstico de la FQ puede ser atribuido a múltiples variables incluyendo diagnóstico tardío, falta de conocimiento apropiado por los médicos y pacientes, la falta y demora de derivaciones de pacientes a los CDFQ, subdiagnóstico, la falta de programas de pesquisa neonatal en muchas regiones y hospitales, dificultad en el acceso a medicaciones, escasez de CDFQ y altos índices de desnutrición.

En un estudio retrospectivo de 1960 a 1989 en 10 países Latinoamericanos se concluyó que la edad mediana al diagnóstico era de 3,72 años y la edad mediana al fallecimiento era de 6,68 años $^{11}$, aunque estudios más recientes de Brasil sugieren mejoría en estos resultados ${ }^{18}$. En el caso específico de Chile la sobrevida actual no alcanza a más de 12 años ${ }^{19}$. En 1991, en un estudio epidemiológico sudamericano en el que participó Chile se dice: "considerando solamente datos chilenos, entre sus resultados destaca la edad promedio de defunción (3,1 años) y el tiempo de sobrevida de $50 \%$ de los pacientes al momento del diagnóstico (4-5 años)"20.

En lo que se refiere a hitos importantes en FQ en Argentina, podemos citar el consenso de tratamiento de $2008^{21}$. No podemos hacer comparaciones con cifras nacionales de sobrevida ya que no están disponibles. Los intercambios de información con un importante CDFQ argentino confirman que no tienen resultados de sobrevida. Es así que nuestra organización presenta características únicas al respecto. En el caso particular de Mendoza, hemos observado un aumento sustancial de la sobrevida entre las cohortes asociado con la organización de un CDFQ de acuerdo con normas internacionales.

El aumento de la sobrevida y el incremento de pacientes de edades mayores, ha presentado nuevos desafíos en lo que se refiere a la transferencia de estos pacientes a centros de adultos $^{22-24}$.

Nuestros hallazgos sugieren que las importantes diferencias en resultados que hay entre el mundo desarrollado y el mundo en desarrollo pueden ser reducidas a través de la organización de CDFQ que provean un mejor cuidado multidisciplinario a los pacientes. La organización de un CDFQ permite el uso racional de normas internacionales de tratamiento, facilita el desarrollo de experiencia clínica y aplicación de tecnologías, y facilita la aplicación sistemática de terapéuticas costosas mejorando así la relación costo-beneficio.

En este trabajo se presentan los resultados de un solo CDFQ. La duplicación de estos resultados en otras áreas del mundo en desarrollo sería un paso importante para mejorar la sobrevida global de la FQ en Latinoamérica. El profundo y desinteresado compromiso de los médicos y el personal del CDFQ ha sido sin duda un factor primordial en nuestras cifras. 
Mantener las fuentes de recursos que lo hagan funcionar es un gran desafío: nuestro CDFQ solicita continuamente que sus partidas presupuestarias sean actualizadas. No debemos aceptar estas diferencias de sobrevida en Latinoamérica como inevitables ya que hemos demostrado que aún con presupuestos acotados, la organización, la normatización y el compromiso personal hacen la diferencia.

Los factores asociados a la mejoría en sobrevida inluyen uso de diferentes medicaciones y procedimientos, como son el uso de los protocolos internacionales, desarrollo de habilidades para inserción de catéteres ${ }^{13}$, uso de bombas de infusión, cambios de antibióticos de acuerdo a resistencias bacterianas, aparición de nuevos antibióticos y variaciones en las dosis ${ }^{14,25}$, gastrostomías para alimentación hipercalórica, entrenamiento de las familias y los pacientes en kinesioterapia respiratoria ${ }^{15}$, el uso de ADNasa y de Tobramicina inhalada ${ }^{16}$, el uso de Bipap como soporte respiratorio, el desarrollo de un sistema de atención domiciliaria estatal y uno privado. Aunque este estudio no fue diseñado para comprobar estas intervenciones, su beneficio ha sido claramente demostrado en otros trabajos científicos y son recomendadas en las normas de la "Cystic $\mathrm{Fi}$ brosis Foundation".

Las debilidadades de este estudio señalan que el diagnóstico tardío fue un factor importante que ha influenciado negativamente las cifras hasta la organización formal del CDFQ en 1989. Actualmente aún hay demoras diagnósticas en pacientes en etapas avanzadas de su enfermedad debido a derivaciones tardías desde otros lugares, lo que determina que la edad media al diagnóstico no haya cambiado. Las cifras de desnutrición dependen mucho de decisiones personales de adolescentes fibroquísticos en los que hemos visto situaciones de hartazgo y hasta "suicida" que determinan que rechacen medicaciones, procedimientos quirúrgicos (gastrostomía) y soporte nutricional avanzado.

En conclusión, el CDFQ de Mendoza ha incrementado sustancialmente la sobrevida en su población. Este estudio demuestra que hay un gran potencial para que los CDFQ debidamente organizados y siguiendo normas internacio- nales, reduzcan las diferencias de sobrevida entre el mundo desarrollado y el subdesarrollado.

\section{Agradecimientos}

Agradecemos a la Cystic Fibrosis Foundation (EEUU) por su apoyo constante. A D.S. Schidlow M.D. en el Hospital St. Christopher's de Philadelphia (EEUU) por enseñanza y práctica en una organización para el tratamiento de la FQ y a nuestro equipo del Hospital H. J. Notti, Mendoza, por sus continuos esfuerzos y compromiso en beneficio de nuestros pacientes.

\section{Referencias}

1.- Cystic Fibrosis Foundation. 2010. Annual report.

2.- Dodge JA, Morison S, Lewis PA, et al: Incidence, population, and survival of cystic fibrosis in the UK, Cystic Fibrosis Survey. Management Committee. Arch Dis Child 1997; 77: 493-6.

3.- Elborn JS, Shale DJ, Britton JR: Cystic Fibrosis: Current survival and population estimates to the year 2000 . Thorax 1991; 46: 881-5.

4.- Lebecque P, Leonard A, De Boeck K, et al: Early referral to cystic fibrosis specialist centre impacts on respiratory outcome. J Cyst Fibros 2009; 8: 26-30.

5.- Flume PA, O'Sullivan BP, Robinson KA, et al: Cystic Fibrosis Pulmonary Guidelines. Chronic Medications for Maintenance of Lung Health. Am J Respir Crit Care Med 2007; 176: 957-69.

6.- Cystic Fibrosis Trust. Antibiotic Treatment for cystic fibrosis. Report of the UK cystic fibrosis Trust Antibiotic Working group. May 2009. Third ed: 4.7.1-4.7.2.

7.- Kalnins D, Durie PR, Pencharz P, et al: Nutritional management of cystic fibrosis patients. Current Opinion in Clinical Nutrition and Metabolic Care 2007; 10: 348-54.

8.- Konstan $M W$ : Dornase Alfa and Progression of Lung Disease in Cystic Fibrosis. Pediatr Pulmonol 2008; 43: S24-S28.

9.- Bender LM, Cotten SW, Willis MS: Kids in America: Newborn Screening for Cystic Fibrosis. Lab Medicine 2011; 42: 595-601.

10.- Tiddens $H A W M$ : Detecting early structural lung damage in cystic fibrosis. Pediatr Pulmonol 2002; 34: 228-31.

11.- Macri CN, Gentile SA, Manterola A: Estudio clínico 
epidemiológico Latinoamericano de la fibrosis quística (mucoviscidosis). Arch Arg Ped 1992; 90: 111-8.

12.- LeGrys VA, Yankaskas JR, Quittel LM, Marshall BC, Mogayzel PJ: Diagnostic Sweat Testing: The Cystic Fibrosis Foundation Guidelines. J Pediatr 2007; 151: 85-9.

13.- Bui S, Babre F, Hauchecorne $S$, et al: Intravenous peripherally-inserted centralcatheters for antibiotic therapy in children with cystic fibrosis. J Cyst Fibros. 2009; 8: 326-31.

14.- McArdle J, Talwalkar J: Macrolides in Cystic Fibrosis. Clin Chest Med 2007; 28: 347-60.

15.- Flume PA, Robinson KA, O'Sullivan BP, et al: Cystic Fibrosis Pulmonary Guidelines: Airway Clearance Therapies. Respir Care 2009; 54: 522-37.

16.- Ramsey BW, Pepe MS, Quan JM, et al: Intermittent administration of inhaled tobramycin in patients with cystic fibrosis. N Engl J Med 1999; 340: 23-30.

17.- Rosenstein BJ, Cutting GR: The diagnosis of Cystic Fibrosis: A consensus statement. J Pediatr 1998; 132: 589-95.

18.- Camargos PAM: Cystic Fibrosis in Brazil: the pediatrician's turn. J Pediatr (Rio J) 2004; 80: 344-6.
19.- Sánchez DI, Pérez MA, Boza ML, et al: Consenso nacional de fibrosis quística. Rev Chil Pediatr 2001; 72 : 356-80.

20.- Cerda LJ, Valdivia CG, Guiraldes CE, Sánchez DI: Mortalidad por fibrosis quística en Chile (1997-2003). Rev Med Chile 2008; 136: 157-62.

21.- Castaños C, Rentería F: Consenso de Fibrosis Quística. Arch Argent Pediatr 2008; (Supl) 106 (5); e01-52.

22.- Lentini E: El problema de la transición de jóvenes adultos con enfermedades especiales a hospitales de adultos. La fibrosis quística como ejemplo. Arch Argent Pediatr 2005; 103: 259-60.

23.- Lentini ER, López Millán A: Transferencia a hospital de adultos de adolescentes con enfermedades crónicas. La fibrosis quística como modelo. En: Herrera O., Quezada A. ed. Enfermedades Respiratorias en Pediatría, Santiago-Buenos Aires: Editorial Mediterráneo, 2012; 529-32.

24.- Yankaskas JR, Marshall BC, Sufian B, Simon RH, Rodman D: Cystic Fibrosis Adult Care. Consensus Conference Report. Chest 2004; 125: 1S- 39S.

25.- Clinical Guidelines: Care of Children with Cystic Fibrosis. Royal Bromptom Hospital 2007. $4^{\text {th }}$ ed: 95-101. 